\title{
The affective foundations of high-reliability organizing
}

\author{
TIMOTHY J. VOGUS ${ }^{1 *}$, NAOMI B. ROTHMAN ${ }^{2}$, KATHLEEN M. SUTCLIFFE ${ }^{3}$ \\ AND KARL E. WEICK ${ }^{3}$ \\ ${ }^{1}$ Vanderbilt Owen Graduate School of Management, Nashville, Tennessee, U.S.A. \\ ${ }^{2}$ College of Business and Economics, Lehigh University, Bethlehem, Pennsylvania, U.S.A. \\ ${ }^{3}$ Stephen M. Ross School of Business, University of Michigan, Ann Arbor, Michigan, U.S.A.
}

\begin{abstract}
Summary The factors that compel individuals to exert the extraordinary effort needed to create high reliability-consistent error-free performance under trying conditions-remain unspecified. Here, we propose that when individuals experience emotional ambivalence and prosocial motivation, it induces the broad thinking and other-orientation that undergird mindful organizing and high reliability. Copyright (C) 2014 John Wiley \& Sons, Ltd.
\end{abstract}

Keywords: high reliability; mindful organizing; prosocial motivation; emotional ambivalence

High-reliability organizations (HROs) such as aircraft-carrier flight decks (Weick \& Roberts, 1993) and nuclear power control rooms (Schulman, 1993) consistently navigate complex, dynamic, and time-pressured conditions in an error-free manner. Research shows highly reliable performance results from mindful organizing-a collective behavioral capability to detect and correct errors and adapt to unexpected events (Weick \& Sutcliffe, 2007). The specific behaviors by which members of an organization enact mindful organizing include discussing potential sources of system failure, questioning assumptions and received wisdom about their work, discussing ways to learn from errors and near misses, and drawing upon and deferring to each other's expertise when needed (Weick \& Sutcliffe, 2007).

Mindful organizing is relevant to organizations of all kinds (Vogus \& Welbourne, 2003). For example, its omission is evident in the recent difficult launch of Healthcare.gov where reports suggest low levels of mindful organizing such as failing to identify sources of problems, question unrealistic assumptions, and defer to developer expertise regarding system readiness (Sun \& Wilson, 2013). A key challenge for mindful organizing is sustaining the high levels of discretionary effort (Levinthal \& Rerup, 2006) and attentiveness it demands. More specifically, maintaining highly reliable performance requires frontline employees to engage in effort that is "beyond the levels attained at psychological and cultural equilibria for human beings" (Schulman, 1993, p. 368). The question that we examine and that remains poorly understood is how do the individuals comprising HROs sustain the extraordinary levels of effort and broad thinking needed to mindfully organize?

The answer we propose is that mindful organizing is more likely when individuals are other-oriented, meaning that they are motivated to work for the benefit of others and are more receptive to others' perspectives and incorporate those perspectives into their work. Two factors make individuals more receptive to others and fuel mindful organizing and highly reliable performance: (1) prosocial motivation, the desire to expend effort to benefit others (Grant, 2008), and (2) emotional ambivalence, the simultaneous experience of positive and negative emotions such as hope and doubt. By orienting individuals toward others-making them sensitive to the needs of others and worried about others' potential failures-prosocial motivation (Grant, 2008) strengthens individuals' orientation

*Correspondence to: Timothy J. Vogus, Vanderbilt Owen Graduate School of Management, 40121 st Avenue South, Nashville, Tennessee 37203, U.S.A. E-mail: timothy.vogus@owen.vanderbilt.edu 
toward the system. Emotional ambivalence enables mindful organizing by making individuals more open to alternative perspectives (Rees, Rothman, Lehavy, \& Sanchez-Burks, 2013) and enhancing the cognitive flexibility (Fong, 2006) needed to anticipate failures and effectively respond to the unexpected. The chaos in HROs is inevitable, but prosocial motivation and emotional ambivalence provide individuals the motivational energy, cognitive flexibility, and judgment ability to function within the chaos.

\section{Prosocial Motivation and Mindful Organizing}

Mindful organizing is a function of individuals acting prosocially-seeing their actions as contributions to a system and subordinating their personal interests to those of the system and its constituents (Weick \& Roberts, 1993). Individuals in HROs must be prosocially motivated to protect their colleagues and stakeholders from the potential harm inherent in their work. Other-orientation increases awareness of others, including who needs help, where useful expertise resides, and how to swiftly mobilize others' expertise when the unexpected occurs (Benner, Tanner, \& Chesla, 1996). When individuals' self-orientation is not redirected toward others, it can lead to poor outcomes. For example, self-orientation produced continued deaths when physicians in the Bristol Royal Infirmary externalized the causes of these incidents as "bad luck" (Weick \& Sutcliffe, 2003).

Prosocial motivation triggers mindful organizing through two processes. First, prosocial motivation increases commitment to those benefitting from one's efforts and stimulates expansive processing of information from and about others as well as personal initiative and discretionary helping behaviors (Grant, 2008). Second, desiring to benefit others leads individuals to consider others' perspectives and synthesize information to help them more effectively (Grant \& Berry, 2011). Thus, high levels of prosocial motivation should increase behaviors of mindful organizing such as questioning assumptions and received wisdom, deferring to the expertise of others, and generating solutions that incorporate others' constraints and interests to solve problems (Grant \& Berry, 2011). These behaviors are improved by the broad thinking and perspective taking fueled by being otheroriented.

There is some support for our arguments. In a qualitative study of an offshore oil rig, Ely \& Meyerson (2010) illustrate how individuals exhibiting proself motivations-showing how unshakable, fearless, and macho they were-were associated with poor safety records and unreliable performance. In response, management implemented a comprehensive safety program that attempted to reshape the culture to be more other-oriented. Specifically, training and signage throughout the rig refocused attention on safety and protecting others from harm. In doing so, the safety initiative aligned people's work with a purpose that connected them to others. The increased prosocial motivation caused individuals to focus more closely on the failures that could harm their peers and learning-oriented behaviors. The oil rig operators moved from macho posturing to demonstrating their concern and affection for others while displaying humility and vulnerability in the name of learning.

\section{Emotional Ambivalence and Mindful Organizing}

Mindful organizing entails balancing proactive search for signs of potential failure with swift responses to unexpected events (Weick \& Sutcliffe, 2007) and requires individuals to be flexible enough to recognize weak signals of failure and accurate enough to intervene correctly. We suggest that emotional ambivalence-the simultaneous experience of contradictory feelings-will be particularly beneficial to HROs in creating balance between confidence and caution.

Ambivalence is often triggered by emotionally complex situations that involve trade-offs (Fong, 2006), such as those observed in HROs (Weick \& Sutcliffe, 2007). We propose that the experience of emotional ambivalence in HROs will induce and sustain mindful organizing via two mechanisms. First, emotional 
ambivalence - $\mathrm{a}$ joint feeling of doubt and hope-signals that an environment is both safe and problematic. This makes individuals more receptive to alternate perspectives, which results in an ability to anticipate the unexpected (Rees, et al., 2013). In other words, complex and dissonant emotional signals should suggest that a change in one's approach toward the environment is necessary and that boundaries should be expanded to considering a broader set of alternatives, in turn enhancing the anticipation and flexibility needed for mindful organizing. Second, because ambivalence creates a sense of unusualness, it causes people to expand their cognitive categories to see more novel associations and think more flexibly (Fong, 2006), which is necessary to respond mindfully to discrepant events. Thus, high levels of emotional ambivalence should lead individuals to engage in behaviors of mindful organizing such as discussing potential sources of failure, questioning assumptions and received wisdom, deferring to the expertise of others, and attempting to solve problems more creatively (Fong, 2006; Rees et al., 2013). These behaviors are improved by the forecasting and flexibility fueled by emotional ambivalence.

Although the experience of emotional ambivalence in any form is likely to foster mindful organizing, it is especially likely when two discrete emotions coexist — doubt and hope. Doubt is the experience of not knowing that energizes inquiry and a search for understanding. More specifically, doubt is a sensation of unease that signals that there is a need to reconsider and revise our understandings (Locke, Golden-Biddle, \& Feldman, 2008). As such, doubt entails physical feelings that suggest there is more work to do, which help sustain mindful organizing (Locke, et al., 2008). HROs transmit and experience doubt, for example, when personnel on aircraft-carrier flight decks recount "war stories" that emphasize how "most positions on this deck were bought in blood" (Weick \& Roberts, 1993). When these accounts are shared, they serve to institutionalize doubt. That is, the stories keep individuals wary of feeling that they have things under control. However, doubt alone can be problematic as it can slow necessary action in the form of "analysis paralysis" or can deteriorate into a debilitating state of fear and paranoia (Weick \& Sutcliffe, 2007).

Mindful organizing is also fueled by hope-an emotion grounded in an appraisal of the challenges in one's environment and one's capabilities for navigating them (Lazarus, 1999). Hope combats the vagaries of unexpected events by labeling threats challenges and instilling a belief in one's ability to be successful (Lazarus, 1999). Thus, hope can propel and sustain mindful organizing in the face of significant obstacles. Golden-Biddle and Correia (2012) describe how hope enabled ThedaCare to implement a model of collaborative care founded on mindful organizing and resulting in highly reliable performance. Specifically, hope provided the confidence that allowed frontline caregivers to entertain new possibilities and pursue inventive action to realize the desired change (Golden-Biddle \& Correia, 2012). Hope alone, however, presents a challenge to mindful organizing and high reliability. Unchecked hope can lead to arrogance (Schulman, 1993) and a willingness to explain away problematic data (Weick \& Sutcliffe, 2003) both of which make it more likely important discrepancies will go undiscussed and overly swift action will occur.

The preceding arguments lead us to propose that it is the simultaneous experience of high levels of doubt and hope that sustains the questioning of existing understandings and provide the capacity for swiftly responding to the unexpected. Benner and colleagues (1996) provide a provocative example of a hospital nursing unit that we re-analyze to illustrate how a highly reliable organization might simultaneously cultivate doubt and hope to nurture mindful organizing. Specifically, on this unit, the most medically challenging patients were assigned to the least experienced nurses. This practice likely causes the most inexperienced nurses to doubt that they can handle such fragile patients, making it easier to disengage from an assessment or a treatment plan and ask for help. On the unit, doubt is potentially buoyed with hope, as the assignment practice makes senior colleagues ready to act as resources and more cognizant of the junior nurses and their needs for coaching and other forms of help.

\section{Future Research and Concluding Thoughts}

We have addressed how individuals in HROs are able to sustain their extraordinary motivation and flexibility in the most trying conditions. In doing so, we proposed that two mechanisms that cause individuals to turn to others- 
prosocial motivation and emotional ambivalence-drive mindful organizing. Linking mindful organizing to prosocial motivation and emotional ambivalence also illustrates one way in which the long-standing divide between mainstream organizational behavior and research on high reliability may be bridged. To further deepen the connections between these researchers we suggest that job design may further enable the prosocial motivation and emotional ambivalence crucial to mindful organizing.

Prosocial motivation and emotional ambivalence are states, and as such, they should respond to situational inducements such as job design. Given the complexity and dynamism in the HRO environment, one might recommend routinizing individual's work to reduce the chaos (Levinthal \& Rerup, 2006). We posit that routinization undermines the complexity and tension needed to trigger prosocial motivation and emotional ambivalence and, in turn, mindful organizing. Creating prosocial motivation relies on experiencing the full impact of one's work (e.g., by experiencing its impact on beneficiaries, Grant \& Berry, 2011). Designing work this way could mean expanding roles to include following up with patients who had been restored to health, property owners whose homes had been saved from a wildland fire, or colleagues who had been protected from harm. Designing jobs in complex and contradictory ways can create the tension fueling emotional ambivalence. For example, wildland firefighters instill such ambivalence when they implement roles that include lookouts and communication links (i.e., hope) as well as escape routes and safety zones (i.e., doubt that a fire is under control, Weick \& Sutcliffe, 2007). Although these job designs hold promise for HROs, they also potentially benefit any organization wherever work is complex and operational reliability critical.

In this Incubator, we proposed how two seemingly unrelated factors-prosocial motivation and emotional ambivalence-cause individuals to become other-oriented in ways that make them more receptive to others' perspectives. It is the humility of focusing on others that provides the motivation and flexibility needed for mindful organizing and highly reliable performance. In this Incubator piece, we further inform the high-reliability literature and also extend research on the effects of prosocial motivation and emotional ambivalence to new organizational domains and behaviors. We hope our initial exploration will inspire a broad array of researchers to pursue and expand these agendas.

\section{Acknowledgements}

We would like to thank Incubator Editor Tom Wright and an anonymous reviewer for comments that improved the clarity and quality of our manuscript. We would also like to thank Ellie Vogus for her enthusiastic support of this project.

\section{Author biographies}

Timothy J. Vogus is an Associate Professor of Management at the Vanderbilt Owen Graduate School of Management. $\mathrm{He}$ received his $\mathrm{PhD}$ from the University of Michigan. His research focuses on the cognitive (mindful organizing), cultural, motivational, and emotional processes through which individuals and workgroups enact highly reliable performance.

Naomi Rothman is an Assistant Professor in the Department of Management at Lehigh University. Prior to Lehigh, Naomi worked as an assistant professor at the University of Illinois Urbana-Champaign. She received her PhD from New York University. Naomi examines the social consequences of emotions, power, and justice in the workplace.

Kathleen M. Sutcliffe is the Gilbert and Ruth Whitaker Professor of Business Administration at the Stephen M. Ross School of Business, University of Michigan. Her research examines how organizations and their members cope with unexpected events, and how complex organizations can be designed to be more reliable and resilient.

Karl Weick is the Rensis Likert Distinguished University Professor Emeritus of Organizational Behavior and Psychology at the University of Michigan. He received his $\mathrm{PhD}$ in social and organizational psychology from Ohio State University. His research interests include sense making and transitions in dynamic events, high-reliability organizations, medical errors, and continuous change. 


\section{References}

Benner, P., Tanner, C. A., \& Chesla, C. A. (1996). Expertise in nursing practice: Caring, clinical judgment, and ethics. New York: Springer Publishing Company.

Ely, R. J., \& Meyerson, D. E. (2010). An organizational approach to undoing gender: The unlikely case of offshore oil platforms. Research in Organizational Behavior, 30, 3-34.

Fong, C. T. (2006). The effects of emotional ambivalence on creativity. Academy of Management Journal, 49(5), 1016-1030.

Golden-Biddle, K., \& Correia, K. (2012). Hope as generative dynamic in transformational change: Creating and sustaining "collaborative care" in the ThedaCare health system. In K. Golden-Biddle \& J. E. Dutton (Eds.), Using a positive lens to explore social change in organizations: Building a theoretical and research foundation (pp. 241-265). New York, NY: Routledge.

Grant, A. M. (2008). Does intrinsic motivation fuel the prosocial fire? Motivational synergy in predicting persistence, performance, and productivity. Journal of Applied Psychology, 93(1), 48-58.

Grant, A. M., \& Berry, J. W. (2011). The necessity of others in the mother of invention: Intrinsic and prosocial motivations, perspective taking, and creativity. Academy of Management Journal, 54(1), 73-96.

Lazarus, R. S. (1999). Hope: An emotion and a vital coping resource against despair. Social Research, 66(2), $653-678$.

Levinthal, D. A., \& Rerup, C. (2006). Crossing an apparent chasm: Bridging mindful and less mindful perspectives on organizational learning. Organization Science, 17(4), 502-513.

Locke, K., Golden-Biddle, K., \& Feldman, M. S. (2008). Making doubt generative: Rethinking the role of doubt in the research process. Organization Science, 19(6), 907-918.

Rees, L., Rothman, N. B., Lehavy, R., \& Sanchez-Burks, J. (2013). The ambivalent mind can be a wise mind: Emotional ambivalence increases judgment accuracy. Journal of Experimental Social Psychology, 49(3), 360-367.

Schulman, P. R. (1993). The negotiated order of organizational reliability. Administration \& Society, 25(3), 353-372.

Sun, L. H., \& Wilson, S. (2013). Health insurance exchange launched despite signs of serious problems [accessed on December 10, 2013]. Available at: http://www.washingtonpost.com/national/health-science/health-insurance-exchange-launched-despitesigns-of-serious-problems/2013/10/21/161a3500-3a85-11e3-b6a9-da62c264f40e_story.html

Vogus, T. J., \& Welbourne, T. M. (2003). Structuring for high reliability: HR practices and mindful processes in reliability-seeking organizations. Journal of Organizational Behavior, 24(7), 877-903.

Weick, K. E., \& Roberts, K. H. (1993). Collective mind in organizations: Heedful interrelating on flight decks. Administrative Science Quarterly, 38(3), 357-381.

Weick, K. E., \& Sutcliffe, K. M. (2003). Hospitals as cultures of entrapment: A reanalysis of the Bristol Royal Infirmary. California Management Review, 45(2), 73-84.

Weick, K. E., \& Sutcliffe, K. M. (2007). Managing the unexpected: Resilient performance in and age of uncertainty, second edition. San Francisco, CA: Jossey-Bass. 